\title{
Assessment of central venous catheter colonization using surveillance culture of withdrawn connectors and insertion site skin
}

\author{
María Jesús Pérez-Granda ${ }^{1,2,3}$, María Guembe ${ }^{3,4^{*}}$, Raquel Cruces $^{3,4}$, José María Barrio ${ }^{1,2}$ and Emilio Bouza ${ }^{1,3,45}$
}

\begin{abstract}
Background: Culture of catheter hubs and skin surrounding the catheter entry site has a negative predictive value for catheter tip colonization. However, manipulation of the hub for culture requires the hubs to be swabbed, introducing potential dislodging of biofilm and subsequent migration of microorganisms. Hubs are usually closed with needleless connectors (NCs), which are replaced regularly. Our objective was to evaluate whether culture of flushed withdrawn NCs is an alternative to hub culture when investigating central venous catheter colonization.

Methods: The study population comprised 49 intensive care unit patients whose central venous catheters had been in place for at least 7 days. Cultures of NCs and skin were obtained weekly.

Results: We included 82 catheters with more than 7 days' indwelling time. The catheter tip colonization rate was $18.3 \%$ (15/82). Analysis of skin and NC cultures revealed a $92.5 \%$ negative predictive value for catheter colonization. Three episodes of catheter-related bloodstream infection (C-RBSI) occurred in patients with colonized catheters.
\end{abstract}

Conclusion: Surveillance of NC and skin cultures could help to identify patients at risk for C-RBSI.

Keywords: Central venous catheters, Surveillance, Skin cultures, Closed needleless connectors, Colonization, Catheter-related bloodstream infection

\section{Background}

Catheter-related bloodstream infection (C-RBSI) is a severe condition with high rates of associated morbidity and mortality $[1,2]$. It occurs after catheter tip colonization by microorganisms progressing along both the inner and outer surface of the catheter [3, 4]. Diagnosis of catheter tip colonization is confirmed by culturing the catheter tip after withdrawal but may be anticipated by conservative methods based on superficial cultures of hubs and the skin surrounding the catheter entry site [5]. Catheter colonization is considered a harbinger of C-RBSI and may be used to identify an at-risk population $[3,4,6-8]$.

\footnotetext{
* Correspondence: mariaguembe@hotmail.com

${ }^{3}$ Instituto de Investigación Sanitaria Gregorio Marañón (IISGM), Dr. Esquerdo, 46, 28007 Madrid, Spain

${ }^{4}$ Department of Clinical Microbiology and Infectious Diseases, Hospital General Universitario Gregorio Marañón, Dr. Esquerdo, 46, 28007 Madrid, Spain Full list of author information is available at the end of the article
}

However, hub culture requires the hubs to be swabbed, introducing potential dislodging of biofilm and subsequent migration of microorganisms $[9,10]$.

At present, most catheter hubs are occluded by closed needleless connectors (NCs), which, according to the manufacturer's instructions, must be replaced regularly [11]. The aim of our study was to evaluate whether culture of flushed withdrawn connectors is an alternative to hub culture when investigating catheter colonization.

\section{Methods \\ Setting}

The major heart surgery intensive care unit (MHS-ICU) in our hospital is a 14-bed post-surgical unit for all adult patients who have undergone a major cardiac surgical procedure. The study population comprised patients who were admitted to the MHS-ICU during the study 
period (12 Jan. 2015 to 31 May 2015) and whose central venous catheter (CVC) had been in place for at least 7 days after insertion. All catheter tips were sent for culture irrespectively of the reason for withdrawal. We excluded patients whose catheter tip had inadvertently not been sent for culture.

\section{Laboratory procedures}

In accordance with the instructions of the manufacturer, NCs (CLAVE systems; ICU Medical, Inc., San Clemente, CA, USA) were changed every 7 days and cultured. Skin cultures were taken simultaneously from the catheter entry site (a swab rubbed into the surface of 1-2 cm around the catheter insertion site) when the $\mathrm{NC}$ was withdrawn and processed following standard semi-quantitative microbiological techniques [5]. All NCs from a single catheter lumen were individually flushed with $100 \mu \mathrm{l}$ of brain-heart infusion and this flush was cultured into a blood agar plate (Figs. 1 and 2). We considered the lumen colonized when at least one culture was positive. The number of NCs cultured varied depending on the number of lumens per catheter (1-5 lumens).

Catheter tips were withdrawn when clinically indicated and cultured immediately by using the roll-plate (Maki) technique or sonication onto a blood agar plate or both [12]. The microorganisms recovered were identified by using standard microbiological methods and matrixassisted laser desorption/ionization-time of flight (MALDITOF) [13]. We used a pre-established protocol to record patient characteristics, underlying diseases, comorbidities, severity of illness scores, and blood culture results at the time of catheter withdrawal.

\section{Definitions}

\section{Catheter tip colonization}

We isolated either at least 15 colony-forming units (CFU) per segment using the semi-quantitative Maki

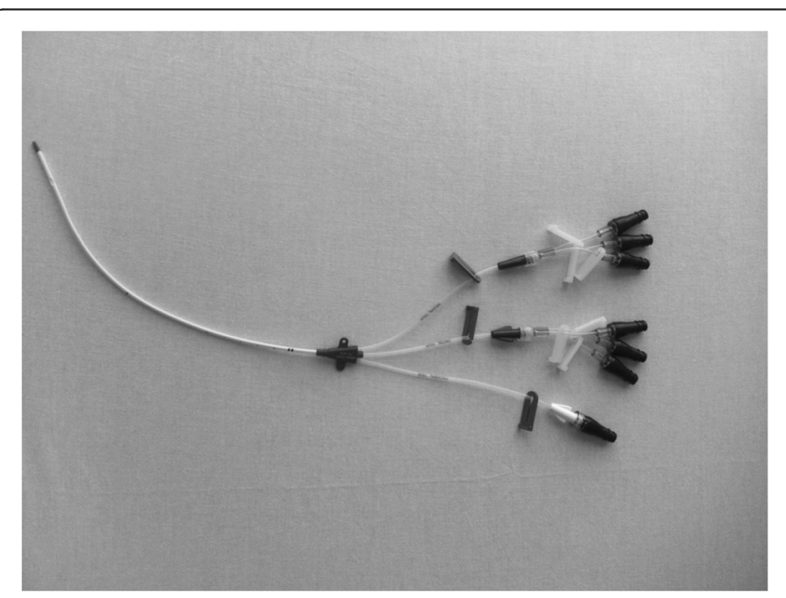

Fig. 1 Central venous catheter

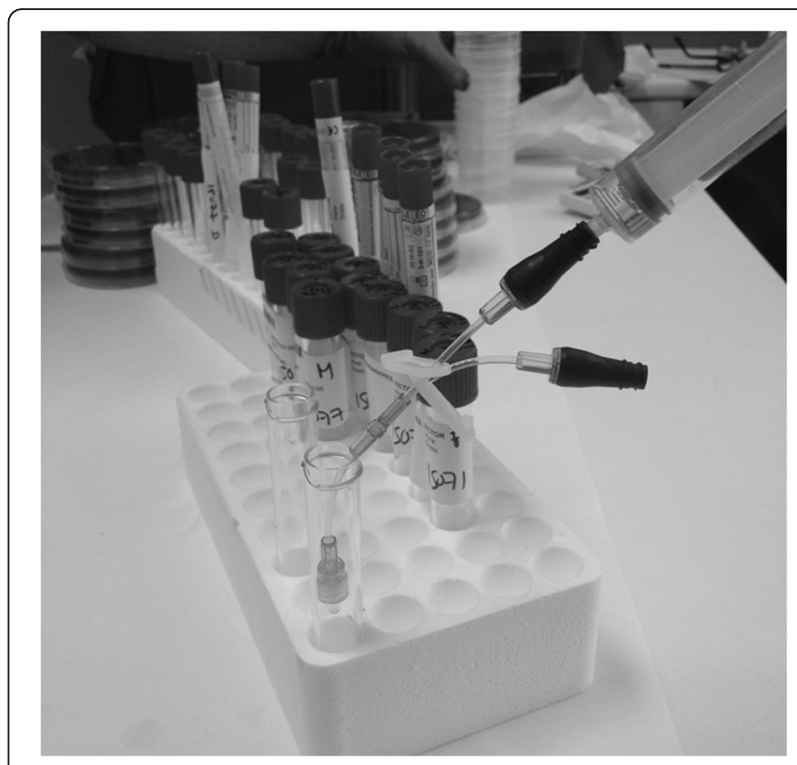

Fig. 2 Laboratory procedure for needleless connector (NC) flushing

technique or at least $100 \mathrm{CFU}$ per segment using the sonication method [5].

\section{Skin colonization}

We isolated at least $15 \mathrm{CFU}$ per plate in the semiquantitative culture [5].

\section{Closed needleless connector colonization}

We isolated at least $10 \mathrm{CFU}$ per connector in at least one connector in the qualitative culture.

\section{Lumen colonization}

Lumen colonization was considered to have occurred when culture of at least one NC from one lumen was positive at any time during surveillance.

\section{C-RBSI}

We considered a C-RBSI episode to be confirmed when the same microorganism was isolated both in peripheral blood cultures (obtained 7 days before or after catheter withdrawal) and from the catheter tip [5]. The gold standard for catheter colonization was positivity of the catheter tip culture by using either the semi-quantitative Maki technique or the quantitative sonication method [5]. To calculate the validity values of skin and $\mathrm{NC}$ cultures for predicting catheter colonization, we used a positive catheter tip with at least $15 \mathrm{CFU}$ per plate of any microorganism as the gold standard.

\section{Statistical analysis}

Continuous variables are expressed as the mean (standard deviation, or SD) or median (interquartile range), and categorical variables as percentages with a $95 \%$ confidence 
interval (CI). Categorical variables were evaluated by using the chi-squared or two-tailed Fisher exact test. Statistical significance was set at a $P$ value of less than 0.05 (two-tailed).

We calculated the validity values of the closed $\mathrm{NC}$ culture by comparing it with the gold standard of colonization. The sensitivity, specificity, and positive and negative predictive values with their $95 \%$ CIs were calculated by using EPIDAT 3.1. Accuracy was defined as the sum of true-positive and true-negative results.

Kaplan-Meier survival curves and the log-rank test were used to compare the time to positivity of the colonization of the first positive skin or NC culture (or both) between colonized and non-colonized catheters. The statistical analysis was performed by using IBM SPSS Statistics for Windows version 21.0 (IBM Corporation, Armonk, NY, USA).

\section{Ethics}

The study was approved by the local ethics committee, and the ethics committee waived the need for informed consent.

\section{Results}

We included 82 catheters with at least 7 days' indwelling time from 49 patients. Mean (SD) age was 64.7 (12.6) years. The main underlying conditions were congestive heart failure (57.1\%), diabetes mellitus (40.8\%), and other diseases $(2.1 \%)$. The overall mean (SD) comorbidity index, Acute Physiology and Chronic Health Evaluation II (APACHE II) score at inclusion, and EuroSCORE were, respectively, 3.4 (4.3), 8.4 (3.0), and 6.9 (2.4). The main reason for catheter withdrawal was end of use (65.9\%), followed by suspicion of infection (24.4\%), and a miscellany of other reasons (9.8\%). We confirmed three episodes of C-RBSI (2.5 episodes per 1000 catheter days). Additional patient and catheter data are shown in Table 1. The crude mortality rate of the selected population under study was $24.4 \%$. We did not find statistically significant differences between the use of parenteral nutrition and catheter colonization $(P=0.34)$.

We collected a total of 656 cultures (82 catheter tips, 148 skin cultures, and $426 \mathrm{NCs}$ ) (Fig. 3). The 82 catheters were evaluated between days 8 and 20 after insertion (median of 11 days). A median of 3 (3-6) NC cultures were performed for each catheter.

The catheter tip colonization rate was $18.3 \%(15 / 82)$. The culture results for the skin and lumens are summarized in Fig. 3. Positive results were detected in $18.2 \%$ $(27 / 148)$ of the skin cultures, and $9.6 \%(41 / 426)$ of the lumen cultures were positive. Positive skin or lumen culture results or both were not detected in $40(48.8 \%)$ of the 82 catheters. In the remaining 42 (51.2\%), the skin or lumens or both were positive at least once.
Table 1 Main characteristics of patients and catheters

\begin{tabular}{|c|c|}
\hline Characteristic & N (\%) \\
\hline \multicolumn{2}{|l|}{ Patients $(n=49)$} \\
\hline Mean (SD) age, years & $64.7(12.6)$ \\
\hline Sex male/female & $30 / 19$ \\
\hline \multicolumn{2}{|l|}{ Underlying conditions } \\
\hline Myocardial infarction & $4(8.2)$ \\
\hline Congestive heart failure & $28(57.1)$ \\
\hline Central nervous system (ACVA) & $10(20.4)$ \\
\hline Chronic obstructive pulmonary disease & $9(18.4)$ \\
\hline Diabetes mellitus & $20(40.8)$ \\
\hline Peptic ulcer disease & $7(14.3)$ \\
\hline Peripheral vascular disease & $3(6.1)$ \\
\hline Renal dysfunction & $9(18.4)$ \\
\hline Mean (SD) EuroSCORE ${ }^{\mathrm{a}}$ & $6.9(2.4)$ \\
\hline Mean (SD) comorbidity index (Charlson criteria) & $3.4(4.3)$ \\
\hline Non-fatal underlying disease (McCabe criteria) & $39(79.6)$ \\
\hline Mean (SD) APACHE II at inclusion & $8.4(3.0)$ \\
\hline Median (IQR) length of ICU stay, days & $13.0(8.0-28.0)$ \\
\hline Crude mortality & $11(24.4)$ \\
\hline \multicolumn{2}{|l|}{ Catheters ( $n=82$ ) } \\
\hline \multicolumn{2}{|l|}{ Type of catheter } \\
\hline Non-tunneled central venous catheter & $62(75.6)$ \\
\hline Guidewire & $20(24.4)$ \\
\hline \multicolumn{2}{|l|}{ Location } \\
\hline Jugular & $78(95.1)$ \\
\hline Subclavian & $4(4.9)$ \\
\hline Total parenteral nutrition & $21(25.6)$ \\
\hline \multicolumn{2}{|l|}{ Reasons for catheter withdrawal } \\
\hline End of use & $54(65.9)$ \\
\hline Suspicion of infection & $20(24.4)$ \\
\hline Other & $8(9.8)$ \\
\hline Median (IQR) indwelling time, days & $11.0(8.0-20.0)$ \\
\hline Total number of catheter days & 1215 \\
\hline Catheter colonization & $15(18.3)$ \\
\hline Catheter colonization, density per 1000 catheter-days & 12.3 \\
\hline C-RBSI episodes & $3(3.7)$ \\
\hline C-RBSI per 1000 catheter days & 2.5 \\
\hline
\end{tabular}

$S D$ standard deviation, IQR interquartile range, ICU intensive care unit, C-RBSI catheter-related bloodstream infection, ACVA acute cerebrovascular accident. aEuroSCORE: European System for Cardiac Operative Risk Evaluation

\section{Prediction of catheter colonization and C-RBSI by skin or NC culture or both}

Analysis of catheter colonization and C-RBSI by considering skin and $\mathrm{NC}$ culture colonization together as a single test showed $80.0 \%$ sensitivity and $92.5 \%$ negative predictive value. In addition, a negative result for all lumen 


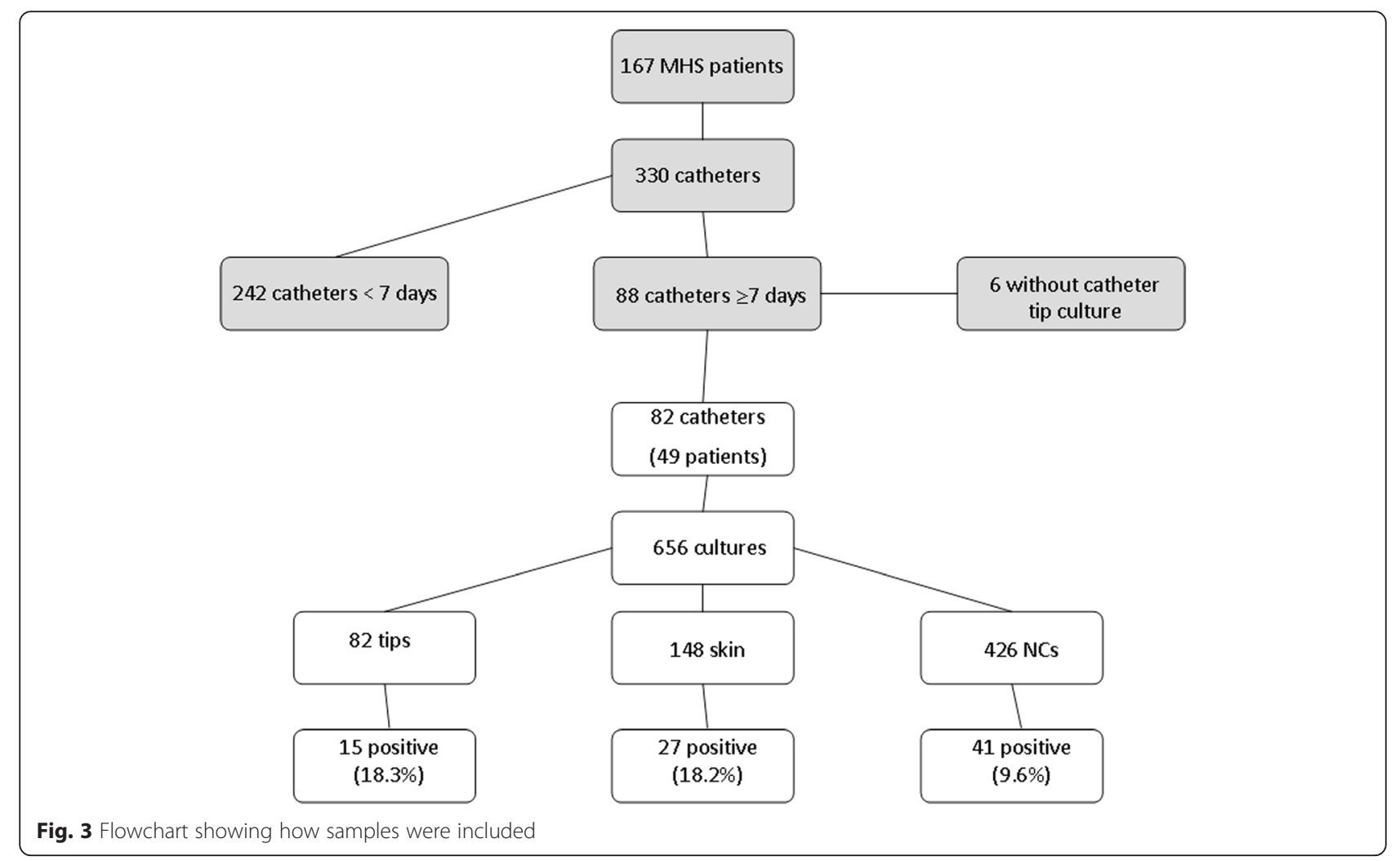

cultures (all NC cultures) had a negative predictive value of $100 \%$ for C-RBSI (Table 2).

The microorganisms isolated from the colonized CVCs are detailed in Table 3.

A Kaplan-Meier analysis showed that the earlier a superficial culture was positive, the greater the chance of catheter tip colonization $(P=0.19)$. Of the 15 colonized catheters, the concordance (identification of genus and species) between superficial cultures (skin or lumens or both) and colonized tips was $73.3 \%$.

\section{Discussion}

Negative cultures from the skin surrounding the catheter entry site and from flushed catheter NCs are good predictors of the absence of catheter tip colonization. In patients with bacteremia, the negativity of skin and NC cultures practically rules out the causal role of the catheter in bloodstream infection.

C-RBSI is a major nosocomial infection with high rates of morbidity and mortality, especially in MHS-ICU patients $[14,15]$. Colonization of the catheter tip is considered a pre-requisite for the development of C-RBSI, which occurs by migration of microorganisms to the catheter tip along the inner or the outer surface $[3,4]$. In clinical practice, more than $50 \%$ of the catheter tips withdrawn with suspected C-RBSI actually prove to be culture-negative in the microbiology department; that is, non-colonized catheters are withdrawn early and unnecessarily [16].
Several authors, including our group, have demonstrated that negative superficial cultures of the skin surrounding the catheter insertion site and catheter hubs ruled out catheter tip colonization in MHS-ICU, oncology, and hemodialysis patients, thus avoiding unnecessary withdrawals of the catheter $[3,6,7]$. Catheter hub cultures are obtained by rubbing swabs on the inside of the hubs and therefore carry a potential risk of dislodging microorganisms [9-11].

Considering that NCs are replaced regularly to decrease the possibility of colonization, we thought that they could be used as an alternative diagnostic method to hub cultures and thus would enable us to avoid unnecessary manipulation. We recently demonstrated that NCs were capable of ruling out catheter tip colonization by culturing their outer surface after sonication [17]. However, we did not assess the yield of the inner surface of NCs combined with skin culture for prediction of catheter colonization and C-RBSI. In the present study, we showed that negative NCs and superficial cultures had a high negative predictive value for catheter colonization and practically ruled out the catheter as the source of bacteremia.

The main limitations of our study were its small sample size, the need to obtain a high number of $\mathrm{NC}$ cultures (with the consequent high workload and costs), and the fact that our results cannot be immediately extrapolated to populations other than MHS-ICU patients. 
Table 2 Validity values of skin and needleless connector cultures for prediction of catheter colonization and catheter-related bloodstream infection

\begin{tabular}{|c|c|c|c|c|c|c|c|c|}
\hline Cultures & $\begin{array}{l}\text { S \% } \\
(95 \% \mathrm{Cl})\end{array}$ & $\begin{array}{l}\text { SP \% } \\
(95 \% \mathrm{Cl})\end{array}$ & $\begin{array}{l}\text { PPV \% } \\
(95 \% \text { Cl) }\end{array}$ & $\begin{array}{l}\text { NPV \% } \\
(95 \% \text { Cl) }\end{array}$ & $\begin{array}{l}\text { Validity index } \\
(95 \% \text { Cl) }\end{array}$ & $\begin{array}{l}\text { Prevalence } \\
(95 \% \mathrm{Cl})\end{array}$ & $\begin{array}{l}\mathrm{LR}^{+} \\
(95 \% \mathrm{Cl})\end{array}$ & $\begin{array}{l}\mathrm{LR}^{-} \\
(95 \% \mathrm{Cl})\end{array}$ \\
\hline \multicolumn{9}{|c|}{ Catheter colonization } \\
\hline \multirow[t]{2}{*}{ Skin + NCs } & 80.0 & 55.2 & 28.6 & 92.5 & 59.8 & 18.3 & 1.79 & 0.36 \\
\hline & $(56.4-100)$ & $(42.6-67.9)$ & (13.7-4434)- & (83.0-100) & $(48.5-71.0)$ & $(9.3-27.3)$ & $(1.24-2.58)$ & $(0.13-1.02)$ \\
\hline \multirow[t]{2}{*}{ Skin } & 66.7 & 82.0 & 45.4 & 91.7 & 79.3 & 18.3 & 3.72 & 0.41 \\
\hline & $(39.5-93.9)$ & (72.2-92.0) & $(22.4-68.5)$ & (83.8-99.5) & $(69.9-88.6)$ & $(9.3-27.3)$ & $(1.99-6.96)$ & $(0.20-0.84)$ \\
\hline \multirow[t]{2}{*}{ NCs } & 40.0 & 67.2 & 21.4 & 83.3 & 62.2 & 18.3 & 1.22 & 0.89 \\
\hline & $(11.9-68.1)$ & $(55.2-79.2)$ & $(4.24-38.4)$ & $(72.5-94.2)$ & $(51.0-73.3)$ & $(9.3-27.3)$ & $(0.60-2.47)$ & $(0.57-1.40)$ \\
\hline \multicolumn{9}{|l|}{ C-RBSI } \\
\hline \multirow[t]{2}{*}{ Skin + NCs } & 100 & 50.6 & 7.1 & 100 & 52.4 & 3.7 & 2.03 & NA \\
\hline & (83.3-100) & $(39.0-62.3)$ & $(0.0-16.1)$ & $(98.7-100)$ & $(41.0-63.9)$ & $(0.0-8.3)$ & $(1.62-2.53)$ & \\
\hline \multirow[t]{2}{*}{ Skin } & 100 & 75.9 & 13.6 & 100 & 76.8 & 3.7 & 4.16 & NA \\
\hline & $(83.3-100)$ & $(65.9-86.0)$ & $(0.0-30.2)$ & $(99.2-100)$ & $(67.0-86.6)$ & $(0.0-8.3)$ & $(2.81-6.15)$ & \\
\hline \multirow[t]{2}{*}{ NCs } & 66.7 & 67.0 & 7.1 & 98.1 & 67.0 & 3.7 & 2.03 & 0.50 \\
\hline & $(0.0-100)$ & $(56.0-78.0)$ & $(0.0-18.5)$ & (93.6-100) & $(56.3-77.8)$ & $(0.0-8.3)$ & $(0.86-4.79)$ & $(0.10-2.48)$ \\
\hline
\end{tabular}

$S$ sensitivity, SP specificity, $P P V$ positive predictive value, $N P V$ negative predictive value, $L R+$ positive likelihood ratio, $L R-$ negative likelihood ratio, $C /$ confidence interval, NA not applicable, NC needleless connector, $C-R B S I$ catheter-related bloodstream infection

Table 3 Microorganisms isolated in colonized catheters

\begin{tabular}{|c|c|c|c|}
\hline Catheter tip & Skin + NC & Skin & NC \\
\hline Staphylococcus epidermidis & Staphylococcus epidermidis & Staphylococcus epidermidis & - \\
\hline Staphylococcus epidermidis & Staphylococcus epidermidis & - & Staphylococcus epidermidis \\
\hline Staphylococcus epidermidis & - & - & - \\
\hline Proteus mirabilis & Proteus mirabilis & Proteus mirabilis & - \\
\hline Proteus mirabilis & Proteus mirabilis & Proteus mirabilis & - \\
\hline \multirow[t]{3}{*}{ Staphylococcus epidermidis } & Staphylococcus epidermidis & Staphylococcus epidermidis & Staphylococcus epidermidis \\
\hline & Staphylococcus haemolyticus & - & Staphylococcus haemolyticus \\
\hline & Staphylococcus chromogenes & - & Staphylococcus chromogenes \\
\hline Staphylococcus epidermidis & Staphylococcus epidermidis & Staphylococcus epidermidis & - \\
\hline Staphylococcus epidermidis & Staphylococcus epidermidis & Staphylococcus epidermidis & Staphylococcus epidermidis \\
\hline Staphylococcus epidermidis & Staphylococcus epidermidis & Staphylococcus epidermidis & - \\
\hline CoNS & - & - & - \\
\hline \multirow[t]{3}{*}{ Staphylococcus epidermidis } & Staphylococcus epidermidis & Staphylococcus epidermidis & Staphylococcus epidermidis \\
\hline & Moraxella osloensis & - & Moraxella osloensis \\
\hline & Klebsiella pneumoniae & Klebsiella pneumoniae & - \\
\hline Candida albicans & Staphylococcus hominis & - & Staphylococcus hominis \\
\hline \multirow[t]{3}{*}{ Staphylococcus epidermidis } & Staphylococcus epidermidis & Staphylococcus epidermidis & - \\
\hline & Staphylococcus aureus & & Staphylococcus aureus \\
\hline & Staphylococcus saprophyticus & & Staphylococcus saprophyticus \\
\hline Staphylococcus epidermidis & Staphylococcus epidermidis & Staphylococcus epidermidis & - \\
\hline Staphylococcus hominis & - & - & - \\
\hline
\end{tabular}


Our findings may be interpreted with caution because of the extrapolation of the microbiological results to the clinical setting. Whether clinicians are supposed to use this information to decide that it is appropriate to maintain a central line that could otherwise be removed or to replace a colonized CVC with a new one is a point that should be assessed in future clinical studies.

Clinical trials are required to verify whether early withdrawal of catheters in patients with positive superficial cultures could contribute to the objective of "zero tolerance" of C-RBSI in ICUs, especially in patients with problems of vascular accessibility, coagulopathy, or severe respiratory disease to avoid the CVC removal and the risk of mechanical complications during the new canalization. In addition, the value of these cultures needs to be assessed in terms of the number of sets of $\mathrm{NCs}$ and catheters obtained in each patient.

\section{Conclusions}

If our data are confirmed by other groups, surveillance of catheter colonization (using NCs and skin cultures) could help to identify patients at risk of C-RBSI. These methods might help identify patients at low risk for C-RBSI.

\section{Key message}

- Closed NCs may serve as a safer alternative diagnostic procedure to predict catheter colonization in MHS-ICU patients.

\begin{abstract}
Abbreviations
CFU: Colony-forming unit; Cl: Confidence interval; CVC: Central venous catheter; C-RBSI: Catheter-related bloodstream infection; ICU: Intensive care unit; MHS-ICU: Major heart surgery intensive care unit; NC: Needleless connector; SD: Standard deviation.
\end{abstract}

\section{Competing interests}

The authors declare that they have no competing interests.

\begin{abstract}
Authors' contributions
MG participated in the study conception and design, carried out the analysis and interpretation of the data, and carried out the manuscript writing. MJP-G participated in the study conception and design, performed the statistical analysis, and was involved in drafting the manuscript. JMB participated in the study conception and helped to draft the manuscript. RC carried out the sample collection and data acquisition and was involved in drafting the manuscript. EB participated in the study conception and revised the manuscript critically for important intellectual content. All authors read and approved the final manuscript.
\end{abstract}

\section{Acknowledgments}

We thank Thomas O'Boyle for his help in the preparation of the manuscript.

\section{Financial support}

The study was supported by the project CP13/00268 (integrated in Plan Estatal I+D+I 2013-1016) and co-financed by ISCIII-Subdirección General de Evaluación y Fomento de la Investigación el Fondo Europeo del Desarrollo Regional (FEDER). María Guembe was supported by grants from the Instituto de Salud Carlos III (Miguel Servet).

\section{Author details}

${ }^{1}$ CIBER Enfermedades Respiratorias-CIBERES (CB06/06/0058), Madrid, Spain. ${ }^{2}$ Cardiac Surgery Postoperative Care Unit, Hospital General Universitario Gregorio Marañón, Madrid, Spain. ${ }^{3}$ Instituto de Investigación Sanitaria Gregorio Marañón (IISGM), Dr. Esquerdo, 46, 28007 Madrid, Spain. ${ }^{4}$ Department of Clinical Microbiology and Infectious Diseases, Hospital General Universitario Gregorio Marañón, Dr. Esquerdo, 46, 28007 Madrid, Spain. ${ }^{5}$ Medicine Department, School of Medicine, Universidad Complutense de Madrid, Pza. Ramón y Cajal, s/n Ciudad Universitaria, 28040 Madrid, Spain.

Received: 27 November 2015 Accepted: 16 January 2016 Published online: 02 February 2016

\section{References}

1. Maki DG, Kluger DM, Crnich CJ. The risk of bloodstream infection in adults with different intravascular devices: a systematic review of 200 published prospective studies. Mayo Clin Proc. 2006;81:1159-71.

2. Palomar M, Alvarez-Lerma F, Riera A, Diaz MT, Torres F, Agra Y, et al. Impact of a national multimodal intervention to prevent catheter-related bloodstream infection in the ICU: the Spanish experience. Crit Care Med. 2013;41:2364-72.

3. Bouza E, Alvarado N, Alcala L, Perez MJ, Rincon C, Munoz P. A randomized and prospective study of 3 procedures for the diagnosis of catheter-related bloodstream infection without catheter withdrawal. Clin Infect Dis. 2007:44:820-6.

4. Cercenado E, Ena J, Rodriguez-Creixems M, Romero I, Bouza E. A conservative procedure for the diagnosis of catheter-related infections. Arch Intern Med. 1990;150:1417-20.

5. Mermel LA, Allon M, Bouza E, Craven DE, Flynn P, O'Grady NP, et al. Clinical practice guidelines for the diagnosis and management of intravascular catheter-related infection: 2009 Update by the Infectious Diseases Society of America. Clin Infect Dis. 2009;49:1-45.

6. Bouza E, Rojas L, Guembe M, Marin M, Anaya F, Luno J, et al. Predictive value of superficial cultures to anticipate tunneled hemodialysis catheterrelated bloodstream infection. Diagn Microbiol Infect Dis. 2014;78:316-9.

7. Guembe M, Martin-Rabadan P, Echenagusia A, Camunez F, RodriguezRosales G, Simo G, et al. Value of superficial cultures for prediction of catheter-related bloodstream infection in long-term catheters: a prospective study. J Clin Microbiol. 2013:51:3025-30.

8. Bouza E, Munoz P, Burillo A, Lopez-Rodriguez J, Fernandez-Perez C, Perez $\mathrm{MJ}$, et al. The challenge of anticipating catheter tip colonization in major heart surgery patients in the intensive care unit: are surface cultures useful? Crit Care Med. 2005:33:1953-60.

9. Raad II. The pathogenesis and prevention of central venous catheter-related infections. Middle East J Anaesthesiol. 1994;12:381-403.

10. Sitges-Serra A, Pi-Suner T, Garces JM, Segura M. Pathogenesis and prevention of catheter-related septicemia. Am J Infect Control. 1995;23:310-6.

11. Bouza E, Munoz P, Lopez-Rodriguez J, Jesus Perez M, Rincon C, Martin Rabadan P, et al. A needleless closed system device (CLAVE) protects from intravascular catheter tip and hub colonization: a prospective randomized study. J Hosp Infect. 2003;54:279-87.

12. Maki DG, Weise CE, Sarafin HW. A semiquantitative culture method for identifying intravenous-catheter-related infection. N Engl J Med. 1977;296:1305-9.

13. O'Hara CM. Manual and automated systems for detection and identification of microorganisms. In: Murray PR, Jorgesen JH, Pfaller MA, Yolken RH, editors. Manual of Clinical Microbiology. Washington, DC.: American Society for Microbiology; 2003. p. 185-207.

14. Perez-Granda MJ, Barrio JM, Munoz P, Hortal J, Rincon C, Rabadan PM, et al. Ethanol lock therapy (E-Lock) in the prevention of catheter-related bloodstream infections (CR-BSI) after major heart surgery (MHS): a randomized clinical trial. PLoS One. 2014;9:e91838.

15. Pittet D, Hulliger S, Auckenthaler R. Intravascular device-related infections in critically ill patients. J Chemother. 1995;7:55-66.

16. Bouza E, Alvarado N, Alcala L, Sanchez-Conde M, Perez MJ, Munoz P, et al. A prospective, randomized, and comparative study of 3 different methods for the diagnosis of intravascular catheter colonization. Clin Infect Dis. 2005:40:1096-100.

17. Guembe M, Perez-Granda MJ, Cruces R, Martin-Rabadan P, Bouza E. Cultures of Needleless Connectors Are Useful for Ruling Out Central Venous Catheter Colonization. J Clin Microbiol. 2015;53:2068-71. 